\title{
Measuring Parkinson's Disease Motor Symptoms with Smartphone-based Drawing Tasks
}

\author{
Elina Kuosmanen \\ Valerii Kan \\ Aku Visuri \\ Center for Ubiquitous Computing, University of Oulu \\ Oulu, Finland \\ firstname.lastname@oulu.fi
}

\begin{abstract}
In Parkinson's disease (PD), patients' motor functionalities are measured by various tests. Spiral drawing is one of the proven techniques for assessing the severity of PD motor symptoms. Commonly the test is performed with pen and paper, with the following visual observation by a clinician. This paper describes the implementation of the digitized version of the spiral drawing test for Android devices. Moreover, the application extends the spiral test and utilizes square-shape drawing accordingly. This artifact was tested in a trial with 8 PD patients and 6 age matching controls. The results have shown the observable difference in performance between PD and non-PD users in drawing accuracy and speed.
\end{abstract}

\section{CCS CONCEPTS}

- Human-centered computing $\rightarrow$ Empirical studies in ubiquitous and mobile computing.

\section{KEYWORDS}

Parkinson's disease; smartphone; logging; empirical evaluation

\section{ACM Reference Format:}

Elina Kuosmanen, Valerii Kan, Aku Visuri, Assam Boudjelthia, Lokmane Krizou, and Denzil Ferreira. 2019. Measuring Parkinson's Disease Motor Symptoms with Smartphone-based Drawing Tasks. In Adjunct Proceedings of the 2019 ACM International foint Conference on Pervasive and Ubiquitous Computing and the 2019 International Symposium on Wearable Computers (UbiComp/ISWC '19 Adjunct), September 9-13, 2019, London, United Kingdom. ACM, New York, NY, USA, 4 pages. https://doi.org/10.1145/3341162.3344833

\section{INTRODUCTION}

Parkinson's disease (PD) is a chronic and progressive neurological disorder impacting heavily on patient's quality of life. The disease influences both physical and mental health. Motor symptoms characterizing PD are tremor, rigidity, slowness of movement, shuffling of gait and a stooped posture [12]. Quantification of motor symptoms usually is performed during clinical visits and is based on

Permission to make digital or hard copies of all or part of this work for personal or classroom use is granted without fee provided that copies are not made or distributed for profit or commercial advantage and that copies bear this notice and the full citation on the first page. Copyrights for components of this work owned by others than ACM must be honored. Abstracting with credit is permitted. To copy otherwise, or republish, to post on servers or to redistribute to lists, requires prior specific permission and/or a fee. Request permissions from permissions@acm.org.

UbiComp/ISWC '19 Adjunct, September 9-13, 2019, London, United Kingdom

(c) 2019 Association for Computing Machinery.

ACM ISBN 978-1-4503-6869-8/19/09 . \$15.00

https://doi.org/10.1145/3341162.3344833

\author{
Assam Boudjelthia \\ Lokmane Krizou \\ Denzil Ferreira \\ Center for Ubiquitous Computing, University of Oulu \\ Oulu, Finland \\ firstname.lastname@oulu.fi
}

subjective measures by a clinician and patient's description [12]. However, this evaluation represents disease status only for a present moment but the severity of symptoms can vary several times per day, when the affect of medication wears off [13]. PD treatment requires customized medication plan for each patient. Finding a way to measure the variation of motor symptoms during the patient's daily routines would help in the customization.

Spiral drawing is a standardized test in the assessment of movement disorders [6]. It is usually conducted with pen and paper and evaluated visually. In particular, spiral drawing is used in diagnosis and evaluation of Parkinson's disease, specifically in distinguishing from another neurological disorder causing tremor, namely essential tremor [7]. It has been shown that the spiral analysis can be conducted digitally, either with a tablet $[6,8,9]$, or with a smartphone $[3,7]$. Also drawing of other shapes, such as lines and circles $[1,9]$ have been used in PD symptom evaluation. These studies target to quantifying and collecting data $[1,6,7]$, classifying PD from healthy $[3,9]$ or supplementing and finding correlation to UPDRS score (Unified Parkinson's Disease Rating Scale [2]).

However, our overarching goal is to develop a set of multiple tools that can be used in daily life for measuring the condition, and it is unclear how the drawing tasks can capture the small changes during the day. In this paper we describe the implementation of smartphone-based drawing exercises for Android OS targeting to PD motor symptom evaluation: bradykinesia, rigidity and tremor. The initial evaluation of the tool is performed with PD patients and healthy controls, and the results represent the opportunity for capturing the effect of PD symptoms by measuring the error rate and the drawing speed.

\section{IMPLEMENTATION AND AN INITIAL EVALUATION}

The created application presents two drawing tasks for the user. Instructions on how to draw the symbol are shown: the spiral is drawn starting from the center, but for the square the user is free to begin drawing wherever he wishes to. After performing the drawing task, performance information about drawing accuracy and time (see Fig. 1) is given. Next, we will describe how the drawing tasks were implemented and the trial procedure.

\subsection{Design of drawing tasks}

The Archimedean spiral can be represented with polar coordinates as $r=a+b \theta$ where $r$ is radius, $a$ is parameter turning the spiral, and $b$ is the distance between successive turnings [10]. We can 

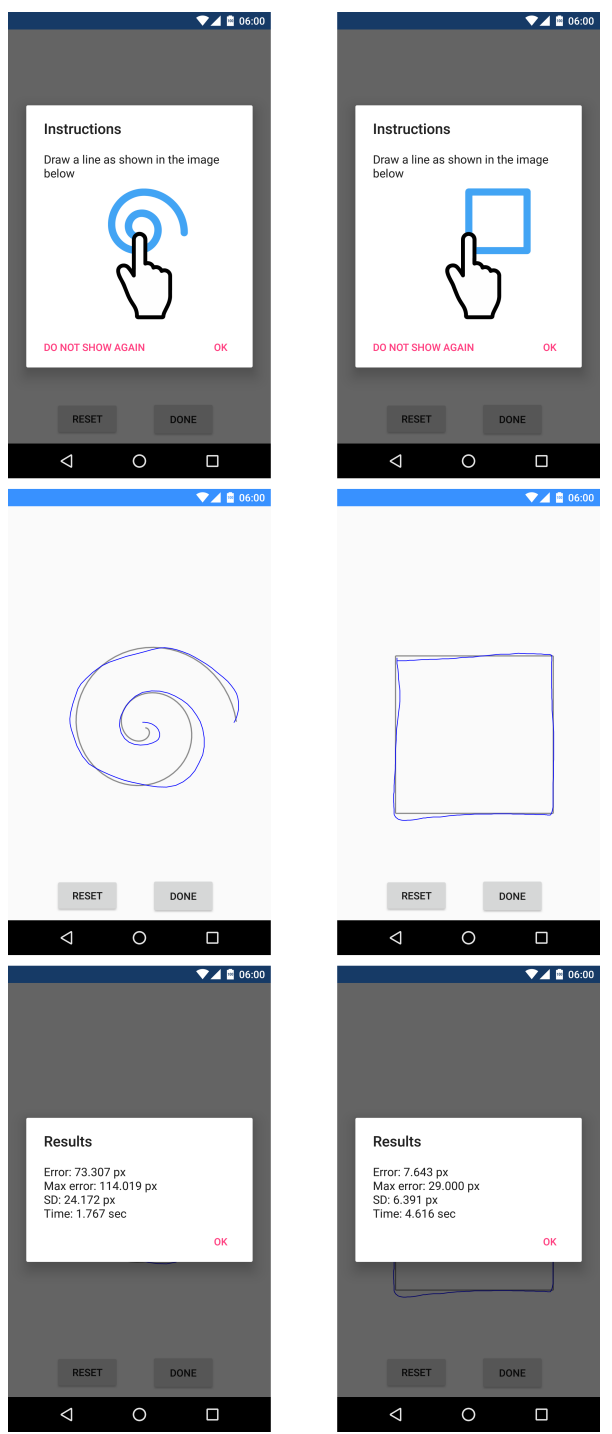

Figure 1: Screen flow of the two drawing tasks - spiral drawing (left) and square drawing (right). From top-down; instructions (top), task (middle), test results (bottom).

use this for calculating the point $(x, y)$ in spiral: $x=r \sin \theta+x_{0}$, $y=r \cos \theta+y_{0}$, where $\left(x_{0}, y_{0}\right)$ are the coordinates of the center of the spiral [10]. In our application, we incrementally increase the $\theta$ from 0 to $4 \pi$, and calculate the coordinates for the spiral with two rounds. Then we combine the points with a line.

A user is asked to follow the given spiral line, starting from the center. In paper version, the user draws the spiral in between of the successive turns of the original line. For enabling the error calculation, the digital version asks the user to follow the line. The place of the finger is recorded, at the frequency enabled by the used device. For each user-drawn $\operatorname{dot}\left(x_{i}, y_{i}\right)$, we can calculate the radius, hence the distance from the center of the original spiral: $r_{i}=$ $\sqrt{\left(y_{i}-y_{0}\right)^{2}+\left(x_{i}-x_{0}\right)^{2}}$. We can calculate the corresponding angle [10]: $\theta_{i}=\arctan \left(\frac{y_{i}-y_{0}}{x_{i}-x_{0}}\right)$. Since $\arctan$ gives values only between 0 and $\pi$, we need to keep track on the full rounds of the spiral, and modify the $\theta_{i}$ accordingly. With this angle we find the closest dot in the spiral model $r_{m}=a+b \theta_{i}$, and count the radial error, similarly as in [10], in each point $e_{i}=\left|r_{i}-r_{m}\right|$. We can then sum the errors, and calculate the average error of the drawing $\operatorname{err}_{a v}=\frac{1}{n} \sum_{i=1}^{n} e_{i}$.

Similar to spiral drawing, we draw the model for the square and ask the user to follow the line. The error calculation for drawing the square is the Euclidean distance to the nearest point in the target square.

After the drawing task, we report the time it took to finish the task, the maximum and average error (in pixel units) with the standard deviation. See Fig. 1 for the screen flow of the tasks.

\subsection{Initial evaluation}

We visited the local Parkinson's association meeting for initial assessment of the implemented tasks. Eight participants with PD (four males and four females), and six age-matching controls (two males and four females) volunteered to our study. They filled a paper questionnaire and conducted the drawing tasks, both task three times. Concretely, we asked participant's age, how long they had suffered from PD, if they had any other conditions affecting to hand's motor abilities, and about their earlier experience on touch screen devices. We also asked if they had conducted spiral drawing earlier. The UPDRS scale [2] is used for general assessment of PD, and the PD patients self-evaluated the part II, "Activities for daily living” [11]. After the drawing we inquired which hand did you use for drawing, whether they are left or right handed, and feedback of the tasks. As a reward we gave the participants a chocolate bar (value $2 €$ ).

\section{RESULTS}

The average age of the participants with PD was 71.5 years and 72.3 in the control group. The time since PD diagnosis varied from one to 22 years, with the average of 8 years. In the control group, no-one reported any condition affecting hands' motor capabilities. Only one participant (with PD) had earlier experience of spiral drawing tasks. Six out of eight PD patients and all in control group owned a touch screen device. All participants were right handed and used their right hand for drawing.

When asked which PD symptoms affect to the hands' motor capabilities, five out of eight reported tremor. Also stiffness, weakness, sudden movements and dyskinesia were mentioned. One PD participant told that non-PD related injuries affect as well, in addition to the PD symptoms. One participant had missed the question.

All participant concluded each drawing task three times, hence we collected 24 spirals and 24 squares drawings from participants with PD, and 18 spirals and 18 squares from controls. In total we collected the data points with timestamps and screen captures from from 84 drawings. All drawings were concluded using the same device.

We asked for feedback of the task with an open-ended question after the task. The feedback given by participants with PD was positive. Half used a word "easy". One said it caused excitement, one described it interesting. One said it went well. Surprisingly, no-one of the controls described drawing as easy. One told it was surprisingly hard, and one told it was challenging. One described 

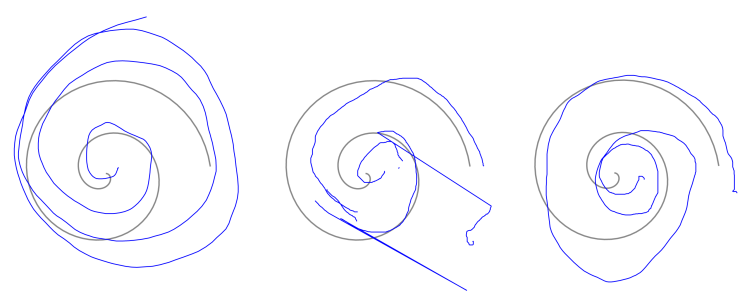

Figure 2: Outliers. Participant NONPD1 drew several rounds extra, PD5's another finger touched the screen causing sharp lines, PD7 had extra round in the beginning of the drawing.

Table 1: Average error in pixels. The PD group has a bigger average error, and the difference is statistically significant.

\begin{tabular}{lcccc}
\hline & PD & Control & t-value & p-value \\
\hline Av. error in spiral & 35.90 & 23.03 & 2.8631 & 0.007591 \\
Av. error in square & 29.83 & 22.49 & 3.9613 & 0.0003193 \\
\hline
\end{tabular}

the touch screen as sensitive, causing error in drawing and requiring focusing. Two participants told the task was interesting.

Based on the screen captures of the drawings, we removed three outliers, see Fig 2. Participant NONPD1 had one drawing where he continued drawing several rounds. That was his first drawing, and we then instructed him when to stop drawing. Participant's PD5 another finger touched accidentally the screen when drawing, that caused unintended lines and a very big error rate. Participant PD7 had one extra round in the beginning of the drawing, and since the error calculation matches the line according to the angle, it caused a very big error rate.

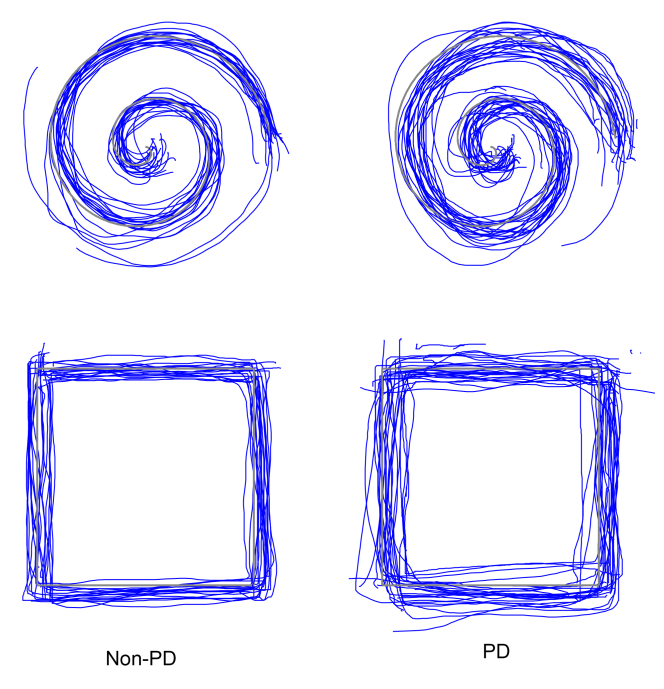

Figure 3: Overlaid drawings of all the participants.

\subsection{Analysis}

We started with a hypothesis that the average error of the PD group is bigger than the control group's average error. With a visual observation from overlaid drawings representation (see Fig 3) we can notice that control group had drawn more smooth shapes. We used t-test, see Table 1, indicating the average error of PD participants is bigger than controls', and the difference is statistically significant.

Figure 4 provides a quartile analysis by R for drawing speed (in pixels/milliseconds). From the graphs of speed and Table 2 we see faster speeds for non-PD cases than for PD patients. Differences can be picked from either median, minimum or average values of the analysis on Table 2 . This suggests that the difference is caused by the PD symptoms, and the digitized drawing tasks are suitable for PD symptom assessment.

We noticed design implications for the error calculation based on the trial. PD5 had problems with another finger hitting in the screen.Another finger causes sharp lines in the other direction, see Fig. 2. Some participants lifted their drawing finger in the middle of task. In spiral drawing, our algorithm detects when we need to add $\pi$ to the angle due to polar coordinates based on difference in the angle between successive points. If the finger has been lifted, we might add $\pi$ erroneously causing mistakes in the error calculation. People lifted their finger more in the square drawing, some
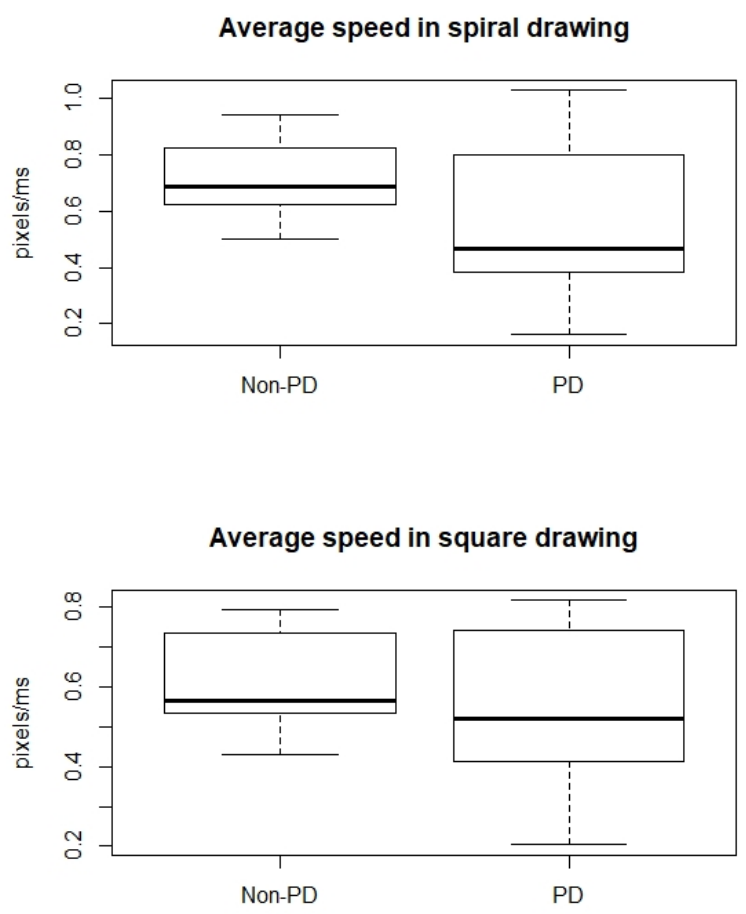

Figure 4: Average speed in drawing tasks in pixels/ms. The average speed is lower and speed variation is bigger in PD group in both tasks. The values can be seen in Table 2 . 
Table 2: PD and Non-PD patients quartile analysis for average speed.

\begin{tabular}{lccccccc}
\hline Groups & $\mathrm{N}$ & $\mathrm{Min}$ & $Q_{1}$ & Median & $Q_{3}$ & Max & Avg. \\
\hline $\begin{array}{l}\text { PD } \\
\text { spiral }\end{array}$ & 22 & 0.16 & 0.38 & 0.47 & 0.80 & 1,03 & 0.56 \\
$\begin{array}{l}\text { Non-PD } \\
\text { spiral }\end{array}$ & 17 & 0.50 & 0.62 & 0.68 & 0.82 & 0.94 & 0.71 \\
$\begin{array}{l}\text { PD } \\
\text { square }\end{array}$ & 24 & 0.20 & 0.41 & 0.52 & 0.74 & 0.82 & 0.54 \\
$\begin{array}{l}\text { Non-PD } \\
\text { square }\end{array}$ & 22 & 0.43 & 0.53 & 0.57 & 0.73 & 0.79 & 0.60 \\
\hline
\end{tabular}

participants did the drawing in parts. PD9 drew the bottom line twice, he did a correction closer to the original line (see Fig. 5). The error calculation of the square matches the drawn line linearly to the closest point in the model, hence the calculation figures a valid error in these cases also.

Due to error calculation mechanism in spiral drawing, finger lifting from the screen should trigger exercise reset. However, this might make the measurement more difficult to conclude: $12 \%(10 / 84)$ of the drawings contained a break; $17 \%$ (8/48) of the PD group's drawings had breaks, while only $5 \%(2 / 36)$ of the control group had these issues. Based on the visual observation during the test, the tremor affects to the motor capabilities of the hand, causing the finger "jumping" in the screen.

If the participant doesn't draw the whole square, like left square in Fig. 5, the missing part is not accounted in the error rate. Hence, we shall reduce the score accordingly if there are missing parts in the drawing.

\section{DISCUSSION AND FUTURE WORK}

This study was a proof of concept for conducting drawing tasks with a smartphone for measuring PD symptoms. The drawing tasks will be a part of a larger set of tools for longitudinal PD symptom assessment; we already have tools for medication logging, measuring the motor symptoms (mainly tremor) with a ball-balancing game, and reporting the daily self-evaluated symptom level [4, 5]. With this combination of tools, we aim to assess the effectiveness of medication.

Similarly to previous studies [6? -9] we saw a difference between the participants with PD and with the age-matching healthy controls in the error rate and the drawing speed. In the trial we saw that the difference was more clear in the spiral drawing task. However, it needs to be investigated if the drawing tasks can be used to measuring daily variation of the symptoms and the effect of the medication, and which symptoms affect to the drawing performance. In future, we will have a study with volunteers whose symptoms are more rigorously identified, to see which of the symptoms the drawing tasks are able to capture. We will extract more parameters from the drawing data, such as the speed change within one drawing. We will combine the drawing data to the data collected by our existing tools[5]. The drawing results will be compared to the ballbalancing game performance metrics, and these combined with the
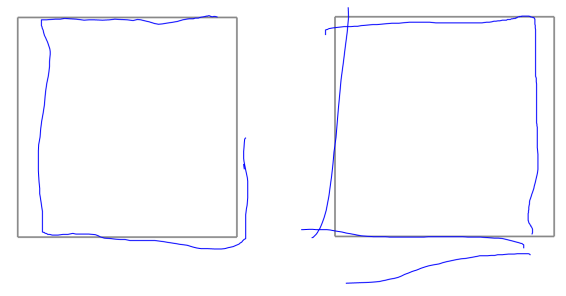

Figure 5: Examples of common errors; Participant PD6 didn't draw a full square. Participant PD9 drew the bottom line twice.

medication logging we can estimate the medication effectiveness and lasting effect.

\section{ACKNOWLEDGMENTS}

This work is partially funded by the Academy of Finland (Grants 313224-STOP, 316253-SENSATE, 320089-SENSATE and 318927-6Genesis Flagship), and a personal research grant awarded by the Finnish Parkinson Foundation.

\section{REFERENCES}

[1] Natalia Dounskaia, Arend WA Van Gemmert, Berta C Leis, and George E Stelmach. 2009. Biased wrist and finger coordination in Parkinsonian patients during performance of graphical tasks. Neuropsychologia 47, 12 (2009), 2504-2514.

[2] SRLE Fahn. 1987. Unified Parkinson's disease rating scale. Recent development in Parkinson's disease (1987).

[3] Ricardo Graça, Rui Sarmento e Castro, and Joao Cevada. 2014. Parkdetect: Early diagnosing parkinson's disease. In Medical Measurements and Applications (MeMeA), 2014 IEEE International Symposium on. IEEE, 1-6.

[4] Valerii Kan. 2018. STOP: a smartphone-based game for Parkinson's disease medication adherence. (2018).

[5] Elina Kuosmanen, Valerii Kan, Aku Visuri, Julio Vega, Yuuki Nishiyama, Anind K Dey, Simon Harper, and Denzil Ferreira. 2018. Mobile-based Monitoring of Parkinson's Disease. In Proceedings of the 17th International Conference on Mobile and Ubiquitous Multimedia. ACM, 441-448.

[6] Seth L Pullman. 1998. Spiral analysis: a new technique for measuring tremor with a digitizing tablet. Movement Disorders 13, S3 (1998), 85-89.

[7] Aleksander Sadikov, Vida Groznik, Jure Žabkar, Martin Možina, Dejan Georgiev, Zvezdan Pirtošek, and Ivan Bratko. 2014. Parkinson Check smart phone app. In Proceedings of the Twenty-first European Conference on Artificial Intelligence. IOS Press, 1213-1214.

[8] Rachel Saunders-Pullman, Carol Derby, Kaili Stanley, Alicia Floyd, Susan Bressman, Richard B Lipton, Amanda Deligtisch, Lawrence Severt, Qiping Yu, Mónica Kurtis, et al. 2008. Validity of spiral analysis in early Parkinson's disease. Movement disorders: official journal of the Movement Disorder Society 23, 4 (2008), 531-537.

[9] Esther J Smits, Antti J Tolonen, Luc Cluitmans, Mark van Gils, Bernard A Conway, Rutger C Zietsma, Klaus L Leenders, and Natasha M Maurits. 2014. Standardized handwriting to assess bradykinesia, micrographia and tremor in Parkinson's disease. PloS one 9, 5 (2014), e97614.

[10] Decho Surangsrirat and Chusak Thanawattano. 2012. Android application for spiral analysis in Parkinson's Disease. In Southeastcon, 2012 Proceedings of IEEE. IEEE, 1-6.

[11] The WE MOVE Clinicians' Guide to Parkinson's disease. 2006. Unified Parkinson's Disease Rating Scale. Retrieved April 27, 2019 from https://img.medscape. com/fullsize/701/816/58977_UPDRS.pdf

[12] Julio Vega. 2016. Monitoring Parkinson's Disease Progression Using Behavioural Inferences, Mobile Devices and Web Technologies. In Proceedings of the 25th International Conference Companion on World Wide Web. International World Wide Web Conferences Steering Committee, 323-327.

[13] Julio Vega, Caroline Jay, Markel Vigo, and Simon Harper. 2017. Unobtrusive Monitoring of Parkinson's Disease Based on Digital Biomarkers of Human Behaviour. In Proceedings of the 19th International ACM SIGACCESS Conference on Computers and Accessibility. ACM, 351-352. 